\title{
A New Look at the Local Solvability Condition of Inhomogeneous Ordinary Differential Equations
}

\author{
Dedicated to Professor Hikosaburo Komatsu on his seventieth birthday
}

By

Shinichi TAJIMA*

\section{$\S 1 . \quad$ Introduction}

Let $P=P(z, d / d z)$ be a linear ordinary differential operator with holomorphic coefficients defined on a neighbourhood $X$ of the origin $O$ in $\mathbb{C}$.

In this paper, we consider the local solvability condition to the inhomogeneous equations $P(z, d / d z) u(z)=f(z)$ in the space $\hat{\mathcal{O}}_{X, O}$ of formal power series at $O$. It is known that straightforward method based on inditial polynomial computation provides only an unsatisfactory answer to this problem, because it does not reveal structure of the equation itself and as a result, for some cases, brute force computations are needed in this direct approach. We propose in this paper an alternative approach with an intention to establish a new effective method to determine the local solvability conditions. For this purpose, we adopt a duality method used by H. Komatsu $([7,8])$ and by H. Komatsu and T. Kawai ([9]) in the study of index theorems and hyperfunction solutions of an ordinary differential equation. We develop a complex variable version of the duality method to show that the local solvability condition can be written in terms of residues. Then upon using the concept of local cohomology and the theory of $D$-modules of one variable, we derive, in a constructive manner, a regular singular holonomic system of ordinary differential equations supported at the origin that completely describes the local solvability condition.

Communicated by T. Kawai. Received November 21, 2005. Revised May 22, 2006.

2000 Mathematics Subject Classification(s): Primary 34M99; Secondary 46F15, 32C36.

* Department of Information Engineering, Faculty of Engineering, Niigata University 8050

Ikarashi Niigata, Niigata 950-2181, Japan.

e-mail: tajima@ie.niigata-u.ac.jp

(c) 2007 Research Institute for Mathematical Sciences, Kyoto University. All rights reserved. 
In section two, we consider local cohomology solutions of the adjoint equation for studying local solvability conditions. We apply the local duality to show in particular that the necessary and sufficient condition for the given function $f$ to be in the space $\operatorname{Im}\left(P, \mathcal{O}_{X, O}\right)$ (resp. $\left.\operatorname{Im}\left(P, \hat{\mathcal{O}}_{X, O}\right)\right)$ can be described in terms of residues, where $\operatorname{Im}\left(P, \mathcal{O}_{X, O}\right)$ (resp. $\left.\operatorname{Im}\left(P, \hat{\mathcal{O}}_{X, O}\right)\right)$ denotes the image space of the map $P: \mathcal{O}_{X, O} \longrightarrow \mathcal{O}_{X, O}$ (resp. $P: \hat{\mathcal{O}}_{X, O} \longrightarrow \hat{\mathcal{O}}_{X, O}$ ) and $\mathcal{O}_{X, O}$ (resp. $\hat{\mathcal{O}}_{X, O}$ ) is the space of convergent power series (resp. formal power series) at the origin $O$.

In section three, we exploit indicial polynomials and develop an effective method for treating algebraic local cohomology solutions of the formal adjoint equation $P^{*} \sigma=0$ associated to $P$. In section four, we apply results given in preceding sections to prove the main results. We first associate to the operator $P$ a regular singular holonomic system of ordinary differential equations supported at the origin that keeps all the necessary information for treating the algebraic local cohomology solutions of the formal adjoint equation $P^{*} \sigma=0$. We emphasize the regular singularity of the resulting system, since the use of the regular singularity is the key point in this approach.

Then we prove, using results of Briançon and Maisonobe ([1]) on $D$ modules of one variable and the duality theorem presented in section two, the main result of this paper which says that local solvability condition in the space $\hat{\mathcal{O}}_{X, O}$ of $P u=f$ can be completely described in terms of the standard basis associated to the regular singular holonomic system.

Some results of this paper have been announced in [14], [15] and [16].

\section{§2. Local Cohomologies and Residues}

We start by recalling some classical results relevant to a local duality ([9], [10], [12]). Let $\mathbb{C}$ be the complex plane with coordinate $z, X$ a neighbourhood of the origin $O$. Let $\mathcal{O}_{X}$ be the sheaf on $X$ of germs of holomorphic functions, $H_{\{O\}}^{1}\left(\mathcal{O}_{X}\right)$ the local cohomology group with support at the origin, which is naturally endowed with a structure of Fréchet-Schwartz topological vector space ([6]). Let $H_{[O]}^{1}\left(\mathcal{O}_{X}\right)$ denote the algebraic local cohomology group, i.e.,

$$
H_{[O]}^{1}\left(\mathcal{O}_{X}\right)=\lim _{k \rightarrow \infty} \operatorname{Ext}_{\mathcal{O}_{X}}^{1}\left(\mathcal{O}_{X} /\left\langle z^{k}\right\rangle, \mathcal{O}_{X}\right) .
$$

The vector space $H_{[O]}^{1}\left(\mathcal{O}_{X}\right)$ has a structure of dual Fréchet-Schwartz topological vector space. Topological vector spaces $H_{\{O\}}^{1}\left(\mathcal{O}_{X}\right)$ and $\mathcal{O}_{X, O}\left(\right.$ resp. $H_{[O]}^{1}\left(\mathcal{O}_{X}\right)$ and $\left.\hat{\mathcal{O}}_{X, O}\right)$ are mutually strong dual vector spaces via local residue pairings, where $\mathcal{O}_{X, O}$ denotes the space of germs of convergent power series (resp. $\hat{\mathcal{O}}_{X, O}$ the space of formal power series) ([4], [5], [9]). 
Let

$$
\operatorname{Res}_{O}\langle,\rangle: \mathcal{O}_{X, O} \times H_{\{O\}}^{1}\left(\mathcal{O}_{X}\right) \longrightarrow \mathbb{C}
$$

denote the residue pairing defined by the local residue

$$
\operatorname{Res}_{O}\langle h, \eta\rangle=\frac{1}{2 \pi i} \oint h(z) \eta(z) d z,
$$

where $h(z) \in \mathcal{O}_{X, O}$ and $\eta(z) \in H_{\{O\}}^{1}\left(\mathcal{O}_{X}\right)$. Let

$$
\operatorname{Res}_{O}\langle,\rangle: \hat{\mathcal{O}}_{X, O} \times H_{[O]}^{1}\left(\mathcal{O}_{X}\right) \longrightarrow \mathbb{C}
$$

also denote the residue pairing between $\hat{\mathcal{O}}_{X, O}$ and $H_{[O]}^{1}\left(\mathcal{O}_{X}\right)$.

Let $P$ be a linear differential operator $P=P(z, d / d z) \in \mathcal{D}_{X, O}$, where $\mathcal{D}_{X, O}$ is the stalk at the origin of the sheaf $\mathcal{D}_{X}$ of rings of linear differential operators. The vector spaces $H_{\{O\}}^{1}\left(\mathcal{O}_{X}\right)$ and $H_{[O]}^{1}\left(\mathcal{O}_{X}\right)$ have a structure of left $\mathcal{D}_{X, O}$-module.

Let $\operatorname{Im}\left(P, \mathcal{O}_{X, O}\right)$ denote the image of the map $P: \mathcal{O}_{X, O} \longrightarrow \mathcal{O}_{X, O}$ and $\operatorname{Im}\left(P, \hat{\mathcal{O}}_{X, O}\right)$ the image of the map $P: \hat{\mathcal{O}}_{X, O} \longrightarrow \hat{\mathcal{O}}_{X, O}$.

Let $\operatorname{Ker}\left(P^{*}, H_{\{O\}}^{1}\left(\mathcal{O}_{X}\right)\right)$ denote the kernel of the mapping

$$
P^{*}: H_{\{O\}}^{1}\left(\mathcal{O}_{X}\right) \rightarrow H_{\{O\}}^{1}\left(\mathcal{O}_{X}\right)
$$

and $\operatorname{Ker}\left(P^{*}, H_{[O]}^{1}\left(\mathcal{O}_{X}\right)\right)$ that of the mapping

$$
P^{*}: H_{[O]}^{1}\left(\mathcal{O}_{X}\right) \rightarrow H_{[O]}^{1}\left(\mathcal{O}_{X}\right),
$$

respectively where $P^{*}$ is the formal adjoint operator of $P$.

Then, we have the following result on the local solvability condition which involves the notion of residues.

Theorem 2.1 ([14]). Let $P$ be a linear differential operator with holomorphic coefficients defined in a neighbourhood of the origin, $P^{*}$ the formal adjoint of $P$.

(i) Let $f$ be a germ at the origin $O$ of holomorphic function. Then, $f \in$ $\operatorname{Im}\left(P, \mathcal{O}_{X, O}\right)$, i.e., the inhomogeneous differential equation $P u=f$ has a solution $u$ in $\mathcal{O}_{X, O}$, if and only if $f \in \mathcal{O}_{X, O}$ satisfies the following condition:

$$
\operatorname{Res}_{O}\langle f, \sigma\rangle=0, \forall \sigma \in \operatorname{Ker}\left(P^{*}, H_{\{O\}}^{1}\left(\mathcal{O}_{X}\right)\right) .
$$

(ii) Let $f$ be a formal power series at the origin. Then, $f \in \operatorname{Im}\left(P, \hat{\mathcal{O}}_{X, O}\right)$, i.e., the inhomogeneous equation $P u=f$ has a formal solution $u$ in $\hat{\mathcal{O}}_{X, O}$, if and only if $f \in \hat{\mathcal{O}}_{X, O}$ satisfies the following condition:

$$
\operatorname{Res}_{O}\langle f, \sigma\rangle=0, \forall \sigma \in \operatorname{Ker}\left(P^{*}, H_{[O]}^{1}\left(\mathcal{O}_{X}\right)\right) .
$$


Proof. Consider the two complexes

$$
\begin{aligned}
& 0 \rightarrow \mathcal{O}_{X, O} \stackrel{P}{\longrightarrow} \mathcal{O}_{X, O} \quad \rightarrow 0, \\
& 0 \leftarrow H_{\{O\}}^{1}\left(\mathcal{O}_{X}\right) \stackrel{P^{*}}{\longleftarrow} H_{\{O\}}^{1}\left(\mathcal{O}_{X}\right) \leftarrow 0 .
\end{aligned}
$$

The kernel spaces and the cokernel spaces associated to these complexes are finite dimensional vector spaces. A standard duality argument, due to [9] and [10], also yields that the vector space $\operatorname{Ker}\left(P^{*}, H_{\{O\}}^{1}\left(\mathcal{O}_{X}\right)\right)$ is the dual space of $\operatorname{Coker}\left(P, \mathcal{O}_{X, O}\right)=\mathcal{O}_{X, O} / \operatorname{Im}\left(P, \mathcal{O}_{X, O}\right)$. Furthermore, since the duality between $\mathcal{O}_{X, O}$ and $H_{\{O\}}^{1}\left(\mathcal{O}_{X}\right)$ is defined by the residue pairing which is compatible with the action of differential operators $([11])$, we have the following non-degenerate pairing (cf. [7])

$$
\operatorname{Res}_{O}\langle,\rangle: \operatorname{Coker}\left(P, \mathcal{O}_{X, O}\right) \times \operatorname{Ker}\left(P^{*}, H_{\{O\}}^{1}\left(\mathcal{O}_{X}\right)\right) \rightarrow \mathbb{C} .
$$

Now, let us consider, for a given $f \in \mathcal{O}_{X, O}$, the inhomogeneous equation $P u=$ $f$. Then the non-degeneracy of the pairing implies that $f \operatorname{lies} \operatorname{in} \operatorname{Im}\left(P, \mathcal{O}_{X, O}\right)$, i.e. $f=0$ in $\operatorname{Coker}\left(P, \mathcal{O}_{X, O}\right)$, if and only if $f$ satisfies the following condition:

$$
\operatorname{Res}_{O}\langle f, \sigma\rangle=0, \forall \sigma \in \operatorname{Ker}\left(P^{*}, H_{\{O\}}^{1}\left(\mathcal{O}_{X}\right)\right),
$$

which completes the proof of (i). Similarly, the non-degeneracy of the pairing (cf. [5])

$$
\operatorname{Res}_{O}\langle,\rangle: \operatorname{Coker}\left(P, \hat{\mathcal{O}}_{X, O}\right) \times \operatorname{Ker}\left(P^{*}, H_{[O]}^{1}\left(\mathcal{O}_{X}\right)\right) \rightarrow \mathbb{C}
$$

yields the second assertion (ii).

Note that the duality theorem for holonomic $D$-modules of partial differential operators has been established by Kashiwara and Kawai ([4], [5]) in a quit general setting i.e., in the context of derived categories, and the notion of residue of several variables has been implicitly utilized. The result above which is an interpretation of the duality for one variable case can also be deduced from Kashiwara-Kawai duality mentioned above. In this paper, we prefer to adopt the duality argument due to Komatsu which is more accessible. Note also that in the statement above, we describe the local solvability condition in terms of residues, because, this is essential for a clear understanding and this important point does not seem to be stated explicitly in the literature. Reader will see that the use of residues provides with us a new effective method for computing the solvability condition.

We give some simple examples for illustration. 
Example 1. Let $P=3 z^{2} \frac{d^{2}}{d z^{2}}+4 z \frac{d}{d z}-4$. The origin is a regular singular point of $P$. The homogeneous equation $P^{*} g=0$ for the formal adjoint operator

$$
P^{*}=3 z^{2} \frac{d^{2}}{d z^{2}}+8 z \frac{d}{d z}-2
$$

has two linearly independent classical solutions $z^{\frac{1}{3}}$ and $\frac{1}{z^{2}}$. Hence we have

$$
\operatorname{Ker}\left(P^{*}, H_{\{O\}}^{1}\left(\mathcal{O}_{X}\right)\right)=\operatorname{Ker}\left(P^{*}, H_{[O]}^{1}\left(\mathcal{O}_{X}\right)\right)=\operatorname{Span}\left\{\left[\frac{1}{z^{2}}\right]\right\} .
$$

Thus, according to Theorem 2.1, if we put $f(z)=c_{0}+c_{1} z+c_{2} z^{2}+\cdots$, the solvability condition to the inhomogeneous equation $P u=f$ is given by

$$
\operatorname{Res}_{O}\left\langle f,\left[\frac{1}{z^{2}}\right]\right\rangle=c_{1}=0 .
$$

Example 2. Let $P=(3 z-1) z^{3} \frac{d^{2}}{d z^{2}}+\left(1-4 z+12 z^{2}\right) z \frac{d}{d z}-2+4 z$. The formal adjoint is

$$
P^{*}=(3 z-1) z^{3} \frac{d^{2}}{d z^{2}}+\left(-1-2 z+12 z^{2}\right) z \frac{d}{d z}-3+6 z .
$$

It follows directly from the index theorem due to Komatsu [8] and Malgrange [10] that the indices

$$
\chi_{P}:=\operatorname{dim} \operatorname{Ker}\left(P, \mathcal{O}_{X, O}\right)-\operatorname{dim} \operatorname{Coker}\left(P, \mathcal{O}_{X, O}\right)
$$

and

$$
\hat{\chi}_{P}:=\operatorname{dim} \operatorname{Ker}\left(P, \hat{\mathcal{O}}_{X, O}\right)-\operatorname{dim} \operatorname{Coker}\left(P, \hat{\mathcal{O}}_{X, O}\right)
$$

of the operator $P$ are equal to -1 and 0 respectively. Since

$$
\operatorname{Ker}\left(P, \mathcal{O}_{X, O}\right)=\operatorname{Ker}\left(P, \hat{\mathcal{O}}_{X, O}\right)=\operatorname{Span}\left\{z^{2}\right\}
$$

we find by virtue of the index formula that

$$
\operatorname{dim} \operatorname{Coker}\left(P, \mathcal{O}_{X, O}\right)=2, \quad \operatorname{dim} \operatorname{Coker}\left(P, \hat{\mathcal{O}}_{X, O}\right)=1 .
$$

In fact, by direct computation we have

$$
\operatorname{Ker}\left(P^{*}, H_{\{O\}}^{1}\left(\mathcal{O}_{X}\right)\right)=\operatorname{Span}\left\{\sigma_{1}, \sigma_{2}\right\},
$$

and

$$
\operatorname{Ker}\left(P^{*}, H_{[O]}^{1}\left(\mathcal{O}_{X}\right)\right)=\operatorname{Span}\left\{\sigma_{1}\right\}
$$


where $\sigma_{1}=\frac{1}{z^{3}} \bmod \mathcal{O}_{X, O}$ and $\sigma_{2}=\exp \left(\frac{1}{z}\right) \bmod \mathcal{O}_{X, O}$.

Let $f=c_{0}+c_{1} z+c_{2} z^{2}+\cdots$ be a convergent power series. Then according to Theorem 2.1, the inhomogeneous equation $P u=f$ has a solution $u$ in $\mathcal{O}_{X, O}$ if and only if

$$
\operatorname{Res}_{O}\left\langle f, \sigma_{1}\right\rangle=\operatorname{Res}_{O}\left\langle f, \sigma_{2}\right\rangle=0 .
$$

Likewise, the solvability condition to the inhomogeneous equation $P u=f$ in the space $\hat{\mathcal{O}}_{X, O}$ is given by $\operatorname{Res} O\left\langle f, \sigma_{1}\right\rangle=0$.

Corollary 2.1 ([14]). Let $\sigma_{1}, \sigma_{2}, \ldots, \sigma_{s}$ be a basis of the local cohomology solution space $\operatorname{Ker}\left(P^{*}, H_{\{O\}}^{1}\left(\mathcal{O}_{X}\right)\right)$ of the formal adjoint equation. Assume that $\sigma_{i}$ has the form

$$
\sigma_{i}=\sum_{j>j_{i}} \frac{c_{i, j}}{z^{j}} \bmod \mathcal{O}_{X, 0}
$$

with $c_{i, j_{i}+1} \neq 0, i=1,2, \ldots, s$, and $j_{1}<j_{2}<\cdots<j_{s}$. Then, $\left\{z^{j_{1}}, z^{j_{2}}, \ldots, z^{j_{s}}\right\}$ constitutes a basis of the vector space $\operatorname{Coker}\left(P, \mathcal{O}_{X, O}\right)$.

Example 3. Let

$P=\left(2-z-2 z^{2}\right) z^{5} \frac{d^{2}}{d z^{2}}+\left(-4+3 z^{2}-10 z^{4}\right) z^{2} \frac{d}{d z}+4+10 z-6 z^{2}-z^{3}+6 z^{4}-6 z^{5}$.

Put

$$
\sigma_{1}=\frac{1}{z^{3}} \exp \left(\frac{1}{z^{2}}\right) \quad \bmod \mathcal{O}_{X, O} \text { and } \sigma_{2}=\frac{1}{z^{5}} \exp \left(\frac{1}{z}\right) \quad \bmod \mathcal{O}_{X, O} .
$$

Then, $\sigma_{1}$ and $\sigma_{2}$ constitute a basis of the local cohomology solution space $\operatorname{Ker}\left(P^{*}, H_{\{O\}}^{1}\left(\mathcal{O}_{X}\right)\right)$. Therefore, $\left\{z^{2}, z^{4}\right\}$ gives rise to a basis of the space $\operatorname{Coker}\left(P, \mathcal{O}_{X, O}\right)$.

\section{$\S 3 . \quad$ Algebraic Local Cohomology Solutions}

In this section, we consider the algebraic local cohomology solutions of the adjoint equation.

coefficients. Put

Let $P=\sum_{i=0}^{n} a_{i}(z) \frac{d^{i}}{d z^{i}}$ be a linear differential operator with holomorphic

$$
e_{P}=\max _{0 \leq i \leq n}\left\{i-v_{O}\left(a_{i}\right)\right\},
$$

where $v_{O}\left(a_{i}\right)$ is the valuation at the origin of $a_{i}(z)$. 
If we put $a_{i}(z)=\sum_{j} a_{i, j} z^{j} \in \mathcal{O}_{X, O}$ with $a_{i, j} \in \mathbb{C}$, then, the initial part $\operatorname{in}(P)$ of the operator $P$ is defined by

$$
\operatorname{in}(P)=\sum_{i-j=e_{P}} a_{i, j} z^{j} \frac{d^{i}}{d z^{i}} .
$$

Recall also that the indicial polynomial $b_{P}(\lambda) \in \mathbb{C}[\lambda]$ of $P$ is defined by

$$
\operatorname{in}(P) z^{\lambda}=b_{P}(\lambda) z^{\lambda-e_{P}} .
$$

Lemma 3.1. Let $P^{*}$ be the formal adjoint of $P$ and $b_{P^{*}}(\lambda)$ the indicial polynomial of $P^{*}$. Then,

(i) $e_{P^{*}}=e_{P}$,

(ii) $\operatorname{in}\left(P^{*}\right)=\operatorname{in}(P)^{*}$,

(iii) $b_{P^{*}}(\lambda)=b_{P}\left(-\lambda-1+e_{P}\right)$.

Let $H_{k}=\left\{\eta \in H_{[O]}^{1}\left(\mathcal{O}_{X}\right) \mid z^{k} \eta=0\right\}$ for a positive integer $k$ and let $H_{k}=\{0\}$ for a non-positive integer $k$.

Lemma 3.2. Let $\sigma$ be an algebraic local cohomology solution of the adjoint equation $P^{*} \sigma=0$. Assume $\sigma \in H_{k}-H_{k-1}\left(i . e ., z^{k} \sigma=0\right.$ and $z^{k-1} \sigma \neq 0$ ) and $k+e_{P}>0$. Then,

$$
b_{P}\left(k-1+e_{P}\right)=0 .
$$

Proof. Since $k+e_{P}>0$, we have

$$
\operatorname{in}\left(P^{*}\right)\left[\frac{1}{z^{k}}\right]=b_{P^{*}}(-k)\left[\frac{1}{z^{k+e_{P}}}\right],
$$

which is equal to $b_{P}\left(k-1+e_{P}\right)\left[\frac{1}{z^{k+e_{P}}}\right]$ by Lemma 3.1. Since the assumption $P^{*} \sigma=0$ implies $\operatorname{in}(P)\left[\frac{1}{z^{k}}\right]=0$, we have $b_{P}\left(k-1+e_{P}\right)=0$.

Let

$$
\begin{aligned}
& B_{P}=\left\{j \in \mathbb{Z} \mid b_{P}(j)=0, j \geq \max \left(e_{P}, 0\right)\right\}, \\
& \Lambda_{P}=\left\{l \in \mathbb{Z} \mid b_{P^{*}}(-l)=0, l \geq \max \left(1,1-e_{P}\right)\right\},
\end{aligned}
$$

and let $\vartheta: B_{P} \longrightarrow \Lambda_{P}$ denote the map defined by $\vartheta(j)=j+1-e_{P}$. Since $\vartheta$ is a bijection, we have $\Lambda_{P}=\vartheta\left(B_{P}\right)$.

\section{Definition 3.1.}

$$
\Gamma=\left\{k \in \mathbb{Z} \mid \exists \sigma \in H_{k}-H_{k-1}, \text { s.t. } P^{*} \sigma=0\right\} .
$$


Put $d_{P}=-e_{P}$ if $e_{P}<0$.

Theorem 3.1. $\quad$ Let $P^{*}$ be the formal adjoint operator of a linear differential operator $P$.

(i) If $e_{P} \geq 0$, then $\Gamma \subset \Lambda_{P}$.

(ii) If $e_{P}<0$, then $\left\{1,2, \ldots, d_{P}\right\} \subset \Gamma \subset\left\{1,2, \ldots, d_{P}\right\} \cup \Lambda_{P}$.

Proof. Since $e_{P^{*}}=e_{P}$, we have $P^{*}\left(H_{k}\right) \subset H_{k+e_{P}}$. Thus, in particular,

$$
H_{d_{P}} \subset \operatorname{Ker}\left(P^{*}, H_{[O]}^{1}\left(\mathcal{O}_{X}\right)\right),
$$

i.e., $\left\{1,2, \ldots, d_{P}\right\} \subset \Gamma$ holds if $e_{P}<0$.

To prove the theorem, it is sufficient to show that $k \in \Lambda_{P}$ provided that $k \in \Gamma$ and $k+e_{P}>0$. Now let $k \in \Gamma$. Suppose $k+e_{P}>0$. Then, by Lemma 3.2, we have $b_{P^{*}}(-k)=b_{P}\left(k-1+e_{P}\right)=0$, which means $k \in \Lambda_{P}$. This completes the proof.

Corollary 3.1. $\quad$ Let $P^{*}$ be the formal adjoint of $P$. Assume that $B_{P}=$ $\emptyset$.

(i) If $e_{P} \geq 0$, then, $\operatorname{Ker}\left(P^{*}, H_{[O]}^{1}\left(\mathcal{O}_{X}\right)\right)=\{0\}$.

(ii) If $e_{P}<0$, then $\operatorname{Ker}\left(P^{*}, H_{[O]}^{1}\left(\mathcal{O}_{X}\right)\right)=H_{d_{P}}$, where $d_{P}=-e_{P}$.

We consider the case $B_{P} \neq \emptyset$. Put $\lambda_{P}=\max \left(\Lambda_{P}\right)$ and $n_{P}=\sharp B_{P}$.

Corollary 3.2. $\quad$ Let $P^{*}$ be the formal adjoint of $P$. Assume that $B_{P} \neq \emptyset$. Then,

$$
\operatorname{Ker}\left(P^{*}, H_{[O]}^{1}\left(\mathcal{O}_{X}\right)\right) \subset H_{\lambda_{P}}
$$

holds. Furthermore, the following holds.

(i) If $e_{P} \geq 0$, then $\operatorname{dim} \operatorname{Ker}\left(P^{*}, H_{[O]}^{1}\left(\mathcal{O}_{X}\right)\right) \leq n_{P}$.

(ii) If $e_{P}<0$, then $\operatorname{dim} \operatorname{Ker}\left(P^{*}, H_{[O]}^{1}\left(\mathcal{O}_{X}\right)\right) \leq n_{P}+d_{P}$,

Example 4. Let $P=z \frac{d^{3}}{d z^{3}}-2 \frac{d^{2}}{d z^{2}}$. Then, $e_{P}=2, \quad b_{P}(\lambda)=\lambda(\lambda-$

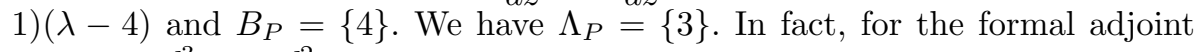
$P^{*}=-z \frac{d^{3}}{d z^{3}}-5 \frac{d^{2}}{d z^{2}}$, we have $e_{P^{*}}=2, b_{P^{*}}(\lambda)=-\lambda(\lambda-1)(\lambda+3)$, which is equal to $b_{P}(-\lambda+1)$. Actually, one can easily verify by direct computation that

$$
\operatorname{Ker}\left(P^{*}, H_{[O]}^{1}\left(\mathcal{O}_{X}\right)\right)=\operatorname{Span}\left(\left[\frac{1}{z^{3}}\right]\right) .
$$


Example 5. Let $P=z^{4} \frac{d}{d z}-z^{3}$. Then, $e_{P}=-3, b_{P}(\lambda)=\lambda-1$ and $B_{P}=\{1\}$. We have $\Lambda_{P}=\{5\}$ and $\{1,2,3\} \subset \Gamma \subset\{1,2,3,5\}$. In fact, we find

$$
\operatorname{Ker}\left(P^{*}, H_{[O]}^{1}\left(\mathcal{O}_{X}\right)\right)=H_{3}+\operatorname{Span}\left(\left[\frac{1}{z^{5}}\right]\right) .
$$

The following result immediately follows from Theorem 2.1 and Corollary 3.1 .

Theorem 3.2. $\quad$ Let $P$ be a linear differential operator. Suppose that $B_{P}=\emptyset$.

(i) If $e_{P} \geq 0$, then $\operatorname{Im}\left(P, \hat{\mathcal{O}}_{X, O}\right)=\hat{\mathcal{O}}_{X, O}$ i.e., for any $f \in \hat{\mathcal{O}}_{X . O}$ the inhomogeneous equation $P u=f$ has a solution $u \in \hat{\mathcal{O}}_{X, O}$.

(ii) If $e_{P}<0$, then $\operatorname{Im}\left(P, \hat{\mathcal{O}}_{X, O}\right)=\left\{f \in \hat{\mathcal{O}}_{X, O} \mid v_{O}(f) \geq d_{P}\right\}$, i.e., the inhomogeneous equation $P u=f$ has a solution $u \in \hat{\mathcal{O}}_{X, O}$ if and only if $v_{O}(f) \geq$ $d_{P}$, where $d_{P}=-e_{P}$.

\section{§4. Regular Singular Holonomic Systems}

In this section, we consider the case where $B_{P} \neq \emptyset$ and investigate the local solvability condition from the viewpoint of algebraic analysis. The key ingredient is the concept of standard bases or Gröbner bases of ideals over the ring of ordinary differential operators ([1], [2], [3]). The use of standard bases allows us to handle, in an explicit way, a regular singular system of the ordinary differential equation itself in question. We refer the reader to [1], [2] for the theory of standard bases in the ring of differential operators.

We define $\mathcal{J}_{P^{*}, k}$ to be the left ideal in $\mathcal{D}_{X, O}$ generated by $P^{*}$ and $z^{k}$;

$$
\mathcal{J}_{P^{*}, k}=\mathcal{D}_{X, O} P^{*}+\mathcal{D}_{X, O} z^{k}, \quad k=1,2, \ldots,
$$

where $z^{k}$ is regarded as a multiplication operator, i.e., a linear differential operator of order zero. Since $z^{k}$ is a regular singular operator, the left $\mathcal{D}_{X, O^{-}}$ module $\mathcal{D}_{X, O} / \mathcal{J}_{P^{*}, k}$ is a regular singular holonomic system supported at the origin $\mathrm{O}$.

It follows directly from the definition that

$$
\operatorname{Hom}_{\mathcal{D}_{X, O}}\left(\mathcal{D}_{X, O} / \mathcal{J}_{P^{*}, k}, H_{[O]}^{1}\left(\mathcal{O}_{X}\right)\right)=\left\{\eta \in H_{k} \mid P^{*} \eta=0\right\} .
$$

We consider the case where $B_{P} \neq \emptyset$. Let $\lambda_{P}=\max \left(B_{P}\right)+1-e_{P}$ and $\mathcal{J}=$ $\mathcal{J}_{P^{*}, \lambda_{P}}$, i.e., $\mathcal{J}=\mathcal{D}_{X, O} P^{*}+\mathcal{D}_{X, O} z^{\lambda_{P}}$. The ideal $\mathcal{J}$ extracts all the necessary information from $P^{*}$ to treat algebraic local cohomology solutions. 
Indeed, we have the following result, which leads a new effective method of handling algebraic local cohomology solutions to the homogeneous equation $P^{*} \sigma=0$.

Lemma 4.1 ([14]). The algebraic local cohomology solution space to the regular singular holonomic system $\mathcal{D}_{X, O} / \mathcal{J}$ is equal to that of the homogeneous equation $P^{*} \sigma=0$, i.e.,

$$
\operatorname{Hom}_{\mathcal{D}_{X, O}}\left(\mathcal{D}_{X, O} / \mathcal{J}, H_{[O]}^{1}\left(\mathcal{O}_{X}\right)\right)=\operatorname{Ker}\left(P^{*}, H_{[O]}^{1}\left(\mathcal{O}_{X}\right)\right)
$$

Proof. Since $\operatorname{Hom}_{\mathcal{D}_{X, O}}\left(\mathcal{D}_{X, O} / \mathcal{J}_{P^{*}, k}, H_{[O]}^{1}\left(\mathcal{O}_{X}\right)\right)=\left\{\eta \in H_{k} \mid P^{*} \eta=0\right\}$, Corollary 3.2 yields the result.

Note that, for any integer $k \geq \lambda_{P}$ we have $\mathcal{J}_{P^{*}, k}=\mathcal{J}$ and $\mathcal{D}_{X, O} / \mathcal{J}_{P^{*}, k}=$ $\mathcal{D}_{X, O} / \mathcal{J}$.

Remark. For the case where $B_{P}=\emptyset$, we define the ideal $\mathcal{J}$ to be $\mathcal{D}_{X, O} P^{*}+\mathcal{D}_{X, O} z^{\lambda_{P}}$ by setting $\lambda_{P}=\max \left(0,-e_{P}\right)$. Thus, $\mathcal{J}=\mathcal{D}_{X, O}$ if $e_{P} \geq 0$. We also have $\mathcal{J}=\mathcal{D}_{X, O} z^{d_{P}}$ if $e_{P}<0$. Indeed, it is easy to see from the definition of $e_{P}$ that if $e_{P}<0$, the formal adjoint $P^{*}$ belongs to the ideal $\mathcal{D}_{X, O} z^{d_{P}}$. Therefore, Lemma 4.1 also holds for this case.

Let us start by considering the simple case where the ideal $\mathcal{J}$ is generated over $\mathcal{D}_{X, 0}$ by a multiplication operator $z^{r_{0}}$. We have the following.

Theorem 4.1. $\quad$ Let $P$ be a differential operator. Suppose that the left ideal $\mathcal{J}$ is generated by a multiplication operator $z^{r_{0}}$. Let $f \in \hat{\mathcal{O}}_{X, O}$. Then the inhomogeneous equation $P u=f$ has a solution $u \in \hat{\mathcal{O}}_{X, O}$ i.e., $f \in \operatorname{Im}\left(P, \hat{\mathcal{O}}_{X, O}\right)$ if and only if $v_{O}(f) \geq r_{0}$.

Proof. Since

$$
\operatorname{Hom}_{\mathcal{D}_{X, O}}\left(\mathcal{D}_{X, O} / \mathcal{D}_{X, 0} z^{r_{0}}, H_{[O]}^{1}\left(\mathcal{O}_{X}\right)\right)=\operatorname{Span}\left\{\left[\frac{1}{z}\right],\left[\frac{1}{z^{2}}\right], \ldots,\left[\frac{1}{z^{r_{0}}}\right]\right\},
$$

Theorem 2.1 and Lemma 4.1 yield the result.

Example 6. Let $P=z^{2} \frac{d}{d z}-z^{2}$. Then, $e_{P}=-1, B_{P}=\{1\}, \Lambda_{P}=$ $\{2\}$ and thus the ideal $\mathcal{J}$ is defined to be $\mathcal{D}_{X, O} P^{*}+\mathcal{D}_{X, O} z^{2}$. Since $P^{*}=$ $-z^{2} \frac{d}{d z}-2 z-z^{2}$ is in $\mathcal{D}_{X, O} z^{2}$, we have $\mathcal{J}=\mathcal{D}_{X, O} z^{2}$. 
Now we address the general case with the help of standard bases that can reveal the structure of the ideal $\mathcal{J}$ in question.

Let $v_{O}(S)$ denote the valuation at the origin $O$ of an ordinary differential operator $S$ which is defined to be the valuation at $O$ of the coefficient function of the highest order term of $S$. We define $\rho \in \mathbb{Z}$ by

$$
\rho=\min \left\{v_{O}(S) \mid S \in \mathcal{J}, S \neq 0\right\},
$$

which is the multiplicity at the origin of the holonomic system $\mathcal{D}_{X, O} / \mathcal{J}$.

Now, let $\left\{R_{0}, R_{1}, \ldots, R_{t}\right\}$ denote the standard basis of the ideal $\mathcal{J}=$ $\mathcal{D}_{X, O} P^{*}+\mathcal{D}_{X, O} z^{\lambda_{P}}$ in the ring $\mathcal{D}_{X, O}$ of differential operators $([1])$. Note that since the ideal $\mathcal{J}$ contains a regular singular differential operator $z^{\lambda_{P}}$, the standard basis $\left\{R_{0}, R_{1}, \ldots, R_{t}\right\}$ consists of regular singular differential operators. We assume that these operators are arranged in such a way that

$$
0=\operatorname{ord}\left(R_{0}\right)<\operatorname{ord}\left(R_{1}\right)<\cdots<\operatorname{ord}\left(R_{t}\right),
$$

where $R_{0}$ stands for a multiplication operator. If we denote by $r_{i}$ the valuation $v_{O}\left(R_{i}\right)$ at the origin of the differential operator $R_{i}$, we have

$$
\lambda_{P} \geq r_{0}>r_{1}>\cdots>r_{t},
$$

and $\rho=r_{0}$ for the case $t=0$ and $\rho=r_{t}$ for the case $t \geq 1$.

In [1], Briançon and Maisonobe proved the following result (cf. [13]).

Lemma $4.2([1])$. Let $\left\{R_{0}, R_{1}, \ldots, R_{t}\right\}$ be the standard basis of the ideal $\mathcal{J}=\mathcal{D}_{X, O} P^{*}+\mathcal{D}_{X, O} z^{\lambda_{P}}$. Then, $\mathcal{J}=\mathcal{D}_{X, O} R_{0}+\mathcal{D}_{X, O} R_{t}$.

Let $R$ denote $R_{t}$ and let $b_{R}(\lambda)$ denote the indicial polynomial of the operator $R$. Let

$$
\begin{aligned}
K_{R} & =\left\{k \in \mathbb{Z} \mid b_{R}(-k)=0\right\} \\
& =\left\{k_{1}, k_{2}, \ldots, k_{m}\right\},
\end{aligned}
$$

where $m=\operatorname{ord}(R)$ and $k_{1}<k_{2}<\cdots<k_{m}$. Since the differential operator $R$ is a member of the standard basis of the ideal $\mathcal{J}$ that defines a regular singular holonomic system supported at the origin, $k_{i}, i=1,2, \ldots, m$ are positive integers belonging to $\Lambda_{P}$, that is, $K_{R} \subset \Lambda_{P}$. We define $\kappa$ to be $k_{m}$, i.e., $\kappa=\max K_{R}$. It is easy to see that, for each $k \in K_{R}$, there exists an algebraic local cohomology solution

$$
\sigma \in \operatorname{Ker}\left(R, H_{[O]}^{1}\left(\mathcal{O}_{X}\right)\right)
$$


s.t. $\sigma \in H_{k}-H_{k-1}$ (see [1]).

Now we define $\Sigma$ to be a set of algebraic local cohomology solutions corresponding to $K_{R}$, i.e.,

$$
\Sigma=\left\{\sigma_{1}, \sigma_{2}, \ldots, \sigma_{m}\right\},
$$

where $R \sigma_{i}=0$ with $\sigma_{i} \in H_{k_{i}}-H_{k_{i}-1}$.

Let $d_{R}=-e_{R}$. Since the differential operator $R$ is a regular singular operator, we have $d_{R}=\rho-m$. Note that $d_{R} \geq d_{P}$.

Proposition 4.1. The following holds.

(i) If $d_{R}=0$, then $\operatorname{Ker}\left(R, H_{[O]}^{1}\left(\mathcal{O}_{X}\right)\right)=\operatorname{Span} \Sigma$.

(ii) If $d_{R}>0$, then $\operatorname{Ker}\left(R, H_{[O]}^{1}\left(\mathcal{O}_{X}\right)\right)=H_{d_{R}}+\operatorname{Span} \Sigma$.

Proof. Since $\sigma_{1}, \ldots, \sigma_{m}$ are linearly independent, $\operatorname{Span} \Sigma$ is a $k$ dimensional vector subspace of the solution space $\operatorname{Ker}\left(R, H_{[O]}^{1}\left(\mathcal{O}_{X}\right)\right)$. Now suppose, $d_{R}=0$. Then $\operatorname{dim} \operatorname{Ker}\left(R, H_{[O]}^{1}\left(\mathcal{O}_{X}\right)\right)=m$, which implies $\operatorname{Ker}\left(R, H_{[O]}^{1}\left(\mathcal{O}_{X}\right)\right)=$ $\operatorname{Span} \Sigma$.

Let us consider the case where $d_{R}>0$. Then, $\min K_{R}>d_{R}$ holds. Hence, for the subspace $H_{d_{R}} \subset \operatorname{Ker}\left(R, H_{[O]}^{1}\left(\mathcal{O}_{X}\right)\right)$, we have $H_{d_{R}} \cap \operatorname{Span} \Sigma=\{0\}$. The equality $\operatorname{dim} \operatorname{Ker}\left(R, H_{[O]}^{1}\left(\mathcal{O}_{X}\right)\right)=m+d_{R}$ implies the assertion.

We are ready to prove the following result which gives an explicit description of the algebraic local cohomology solutions to the homogeneous formal adjoint equation $P^{*} \sigma=0$.

Theorem 4.2. Let $K_{R}=\left\{k_{1}, k_{2}, \ldots, k_{m}\right\}$ and $\Sigma$ a set of algebraic local cohomology solutions corresponding to $K_{R}$. Then, the following holds.

(i) Suppose that $d_{R}=0$. Then,

$$
\Gamma=K_{R} \text { and } \operatorname{Ker}\left(P^{*}, H_{[O]}^{1}\left(\mathcal{O}_{X}\right)\right)=\operatorname{Span} \Sigma .
$$

(ii) Suppose that $d_{R}>0$. Then,

$$
\Gamma=\left\{1,2, \ldots, d_{R}\right\} \cup K_{R} \text { and } \operatorname{Ker}\left(P^{*}, H_{[O]}^{1}\left(\mathcal{O}_{X}\right)\right)=H_{d_{R}}+\operatorname{Span} \Sigma .
$$

Proof. Since $r_{0}=\kappa$, Lemma 4.2 yields

$$
\operatorname{Ker}\left(R, H_{[O]}^{1}\left(\mathcal{O}_{X}\right)\right)=\operatorname{Hom}_{\mathcal{D}_{X, O}}\left(\mathcal{D}_{X, O} / \mathcal{J}, H_{[O]}^{1}\left(\mathcal{O}_{X}\right)\right),
$$

which is equal to $\operatorname{Ker}\left(P^{*}, H_{[O]}^{1}\left(\mathcal{O}_{X}\right)\right)$ by Lemma 4.1. Hence, Proposition 4.1 implies the result. 


\section{Corollary 4.1.}

$$
\operatorname{dim} \operatorname{Ker}\left(P^{*}, H_{[O]}^{1}\left(\mathcal{O}_{X}\right)\right)=m+d_{R}
$$

Example 7. Put $P=\left(3+z^{3}\right) z^{2} \frac{d^{2}}{d z^{2}}+\left(-12+5 z^{3}\right) z \frac{d}{d z}+12+4 z^{3}$. Then, $e_{P}=0$. Since the indicial equation of $P$ at the origin is $(\lambda-1)(\lambda-4)=0$, we have $\Lambda_{P}=\{2,5\}$. The standard basis of the ideal $\mathcal{J}=\mathcal{D}_{X, O} P^{*}+\mathcal{D}_{X, O} z^{5}$ is given by $\left\{z^{2}, z \frac{d}{d z}+2\right\}$. Solving $R \sigma=0$, with $R=z \frac{d}{d z}+2$, we find

$$
\operatorname{Ker}\left(R, H_{[O]}^{1}\left(\mathcal{O}_{X}\right)\right)=\operatorname{Span}\left\{\left[\frac{1}{z^{2}}\right]\right\} .
$$

Note that the homogeneous equation $P^{*} g=0$ has two linearly independent classical solutions $\frac{1}{z^{2}}$ and $\frac{1}{z^{2}} \log z-\frac{1}{z^{5}}$, whereas, by Theorem 4.2 we have

$$
\operatorname{Ker}\left(P^{*}, H_{[O]}^{1}\left(\mathcal{O}_{X}\right)\right)=\operatorname{Span}\left\{\left[\frac{1}{z^{2}}\right]\right\} .
$$

Example 8. Let $P$ be a second order linear ordinary differential operator of the form

$$
\begin{aligned}
\left(3-5 z^{2}-4 z^{7}+2 z^{9}\right) z^{2} \frac{d^{2}}{d z^{2}}+\left(-12-10 z^{2}-68 z^{7}+49 z^{9}\right) z \frac{d}{d z} \\
+12+30 z^{2}-240 z^{7}+240 z^{9} .
\end{aligned}
$$

Then, $e_{P}=0$. The largest non-negative root of the indicial equation $\lambda^{2}-5 \lambda+$ $4=0$ is equal to 4 . We set $\mathcal{J}=\mathcal{D}_{X, O} P^{*}+\mathcal{D}_{X, O} z^{5}$. The standard basis of the ideal $\mathcal{J}$ is $\left\{R_{0}, R_{1}, R_{2}\right\}$, where

$$
R_{0}=z^{5}, R_{1}=z^{3} \frac{d}{d z}+5 z^{2}, R_{2}=z^{2} \frac{d^{2}}{d z^{2}}+8 z \frac{d}{d z}+10 .
$$

Thus $K_{R}=\{2,5\}$. By direct computation, we have $\operatorname{Ker}\left(R, H_{[O]}^{1}\left(\mathcal{O}_{X}\right)\right)=$ $\operatorname{Span}\left\{\left[\frac{1}{z^{2}}\right],\left[\frac{1}{z^{5}}\right]\right\}$. Therefore, according to Theorem 4.2, we have

$$
\operatorname{Ker}\left(P^{*}, H_{[O]}^{1}\left(\mathcal{O}_{X}\right)\right)=\operatorname{Span}\left\{\left[\frac{1}{z^{2}}\right],\left[\frac{1}{z^{5}}\right]\right\} .
$$

Note that we have used the computer algebra system kan/sm1 ([17]) for algebraic analysis developed by N. Takayama to compute standard bases. 
Now we return to the main subject of this paper and consider the local solvability condition of the inhomogeneous ordinary differential equation $P u=$ $f$, where $f, u \in \hat{\mathcal{O}}_{X, O}$. Let $\left\{R_{0}, R_{1}, \ldots, R_{t}\right\}$ be the standard basis of the ideal $\mathcal{J}$. We assume that $t \geq 1$. Let $R=R_{t}, K_{R}=\left\{k_{1}, k_{2}, \ldots, k_{m}\right\}$ with $k_{1}<k_{2}<$ $\cdots<k_{m}$ and $\Sigma=\left\{\sigma_{1}, \sigma_{2}, \ldots, \sigma_{m}\right\} \subset \operatorname{Ker}\left(P^{*}, H_{[O]}^{1}\left(\mathcal{O}_{X}\right)\right)$, where

$$
\sigma_{i} \in \operatorname{Ker}\left(R_{t}, H_{[O]}^{1}\left(\mathcal{O}_{X}\right)\right)
$$

s.t. $\sigma_{i} \in H_{k_{i}}-H_{k_{i}-1}, i=1,2, \ldots, m$.

We have the following.

Theorem 4.3. $\quad$ Let $P$ be a linear differential operator with holomorphic coefficients defined in a neighbourhood of the origin. Assume that $B_{P} \neq 0$. Let $f$ be a formal power series at the origin.

(i) Assume that $d_{R}=0$. Then, $f \in \operatorname{Im}\left(P, \hat{\mathcal{O}}_{X, O}\right)$, i.e., the inhomogeneous equation $P u=f$ has a solution $u \in \hat{\mathcal{O}}_{X, O}$ if and only if

$$
\operatorname{Res}_{O}\left\langle f, \sigma_{i}\right\rangle=0, i=1,2, \ldots, m \text {. }
$$

(ii) Assume that $d_{R}>0$. Then, $f \in \operatorname{Im}\left(P, \hat{\mathcal{O}}_{X, O}\right)$, if and only if

$$
v_{O}(f) \geq d_{R} \quad \text { and } \quad \operatorname{Res}_{O}\left\langle f, \sigma_{i}\right\rangle=0, i=1,2, \ldots, m .
$$

Proof. Theorem 2.1 and Theorem 4.2 yield the result.

The result above implies in particular that the computation of the solvability conditions can be reduced to that of the standard basis of the ideal $\mathcal{J}$ in $\mathcal{D}_{X, O}$.

Example 9. Let

$$
P=\left(8+2 z^{3}-z^{5}\right) z^{2} \frac{d^{2}}{d z^{2}}+\left(-48+12 z^{3}-9 z^{6}\right) z \frac{d}{d z}+96-3 z^{6} .
$$

Then, $e_{P}=0, b_{P}(\lambda)=8\left(\lambda^{2}-7 \lambda+12\right), B_{P}=\{3,4\}$ and thus $\lambda_{P}=5$. It is easy to verify that $\left\{z^{5}, 2 z^{2} \frac{d^{2}}{d z^{2}}+\left(20 z-3 z^{3}\right) \frac{d}{d z}+40-12 x^{3}\right\}$ is the standard basis of $\mathcal{J}=\mathcal{D}_{X, O} P^{*}+\mathcal{D}_{X, O} z^{5}$. We have

$$
\Sigma=\left\{\left[\frac{1}{z^{4}}\right],\left[\frac{4}{z^{5}}\right]-\left[\frac{1}{z^{2}}\right]\right\} .
$$

Now let $f \in \hat{\mathcal{O}}_{X, O}$. Then, $f \in \operatorname{Im}\left(P, \hat{\mathcal{O}}_{X, O}\right)$ if and only if

$$
\operatorname{Res}_{O}\left\langle f,\left[\frac{1}{z^{4}}\right]\right\rangle=\operatorname{Res}_{O}\left\langle f,\left[\frac{4}{z^{5}}\right]-\left[\frac{1}{z^{2}}\right]\right\rangle=0 .
$$


Corollary 4.2. Let $f \in \hat{\mathcal{O}}_{X, O}$. If $v_{O}(f) \geq \kappa$, then $f \in \operatorname{Im}\left(P, \hat{\mathcal{O}}_{X, O}\right)$.

Corollary 4.3. $\quad$ Let $P$ be a linear differential operator with holomorphic coefficients defined in a neighbourhood of the origin. Let $K_{R}=\left\{k_{1}, k_{2}, \ldots, k_{m}\right\}$. (i) Assume that $d_{R}=0$. Then, $z^{k_{1}-1}, z^{k_{2}-1}, \ldots, z^{k_{m}-1}$ constitute a basis of $\operatorname{Coker}\left(P, \hat{\mathcal{O}}_{X, O}\right)$.

(ii) Assume that $d_{R}>0$. Then, $1, z^{1}, \ldots, z^{d_{R}-1}, z^{k_{1}-1}, z^{k_{2}-1}, \ldots, z^{k_{m}-1}$ constitute a basis of $\operatorname{Coker}\left(P, \hat{\mathcal{O}}_{X, O}\right)$.

Since, the multiplicity $\rho$ at the origin of the holonomic system $\mathcal{D}_{X, O} / \mathcal{J}$ defined to be

$$
\rho=\min \left\{v_{O}(S) \mid S \in \mathcal{J}, S \neq 0\right\}
$$

is equal to $d_{R}+m$ by Proposition 4.1, we have the following.

\section{Corollary 4.4.}

$$
\operatorname{dim} \operatorname{Coker}\left(P, \hat{\mathcal{O}}_{X, O}\right)=\rho
$$

Proof. Since $\operatorname{dim} \operatorname{Coker}\left(P, \hat{\mathcal{O}}_{X, O}\right)=\operatorname{dim} \operatorname{Ker}\left(P^{*}, H_{[O]}^{1}\left(\mathcal{O}_{X}\right)\right)$, which is equal to $\operatorname{dim} \operatorname{Ker}\left(R, H_{[O]}^{1}\left(\mathcal{O}_{X}\right)\right)$, the equality $\rho=d_{R}+m$ implies the result.

Let us denote by $L$ the formal adjoint of the operator $R$ and consider the image of the map:

$$
L: \hat{\mathcal{O}}_{X, O} \longrightarrow \hat{\mathcal{O}}_{X, O}
$$

We have the following.

Proposition 4.2.

$$
\operatorname{Im}\left(P, \hat{\mathcal{O}}_{X, O}\right)=\operatorname{Im}\left(L, \hat{\mathcal{O}}_{X, O}\right)
$$

Proof. Let $f \in \hat{\mathcal{O}}_{X, O}$. Since $L^{*}=R, f \in \operatorname{Im}\left(L, \hat{\mathcal{O}}_{X, O}\right)$ if and only if

$$
\operatorname{Res}_{O}\langle f, \sigma\rangle=0, \forall \sigma \in \operatorname{Ker}\left(R, H_{[O]}^{1}\left(\mathcal{O}_{X}\right)\right)
$$

or, equivalently,

$$
\operatorname{Res}_{O}\langle f, \sigma\rangle=0, \forall \sigma \in \operatorname{Ker}\left(P^{*}, H_{[O]}^{1}\left(\mathcal{O}_{X}\right)\right) .
$$

Hence, $f \in \operatorname{Im}\left(L, \hat{\mathcal{O}}_{X, O}\right)$ if and only if $f \in \operatorname{Im}\left(P, \hat{\mathcal{O}}_{X, O}\right)$, by Theorem 4.1.

Let $V_{k}$ denote the space

$$
V_{k}=\left\{g \in \hat{\mathcal{O}}_{X, O} \mid v_{O}(g) \geq k\right\} .
$$


Note that, from $d_{L}=d_{R}$, we have $L\left(V_{k}\right) \subset V_{k+d_{R}}$.

It is easy to see that the set $B_{L}$ associated to the operator $L$ is given by

$$
B_{L}=\left\{j \in \mathbb{Z} \mid j+1+d_{R} \in K_{R}\right\} .
$$

Let $I=\left\{i \in \mathbb{Z} \mid 0 \leq i<\kappa-d_{R}, i \notin B_{L}\right\}$, where $\kappa=\max K_{R}$.

\section{Lemma 4.3.}

(i) $L z^{i}, i \in I$ are linearly independent.

(ii) $L z^{i} \notin V_{\kappa}$ for $i \in I$.

Then we arrive at the following result.

Theorem 4.4. Let $P$ be a linear differential operator. Then,

$$
\operatorname{Im}\left(P, \hat{\mathcal{O}}_{X, O}\right)=\operatorname{Span}\left\{L z^{i} \mid i \in I\right\}+V_{r_{0}} .
$$

Proof. Put $W=\operatorname{Span}\left\{L z^{i} \mid i \in I\right\}+V_{r_{0}}$. We have $W \subset \operatorname{Im}\left(L, \hat{\mathcal{O}}_{X, O}\right)$. Since $\# I=\kappa-d_{R}-m$ and $\kappa=r_{0}$, we have $\operatorname{dim} \hat{\mathcal{O}}_{X, O} / W=d_{R}+m$, which is equal to $\operatorname{dim} \operatorname{Coker}\left(L, \hat{\mathcal{O}}_{X, O}\right)$. Hence, we have $W=\operatorname{Im}\left(L, \hat{\mathcal{O}}_{X, O}\right)$. Therefore, Proposition 4.2 completes the proof.

Example 10. Let $P=\left(3+z^{3}\right) z^{2} \frac{d^{2}}{d z^{2}}+\left(-12+5 z^{3}\right) z \frac{d}{d z}+12+4 z^{3}$ as in Example 7. Then, $\left\{z^{2}, z \frac{d}{d z}+2\right\}$ is the standard basis of $\mathcal{J}$. Thus, $d_{R}=$ $0, K_{R}=\{2\}$ with $R=z \frac{d}{d z}+2$. Hence, $L=-z \frac{d}{d z}+1, B_{L}=\{1\}$ and $I=\{0\}$. Since $L 1=1$, Theorem 4.4 implies

$$
\operatorname{Im}\left(P, \hat{\mathcal{O}}_{X, O}\right)=\operatorname{Span}\{1\}+V_{2} .
$$

\section{References}

[1] J. Briançon and $\mathrm{Ph}$. Maisonobe, Idéaux de germes d'opérateurs différentiels à une variable, Enseign. Math. (2) 30 (1984), no. 1-2, 7-38.

[2] F. Castro, Calculs effectifs pour les idéaux d'opérateurs différentiels, in Géométrie algébrique et applications, III (La Rábida, 1984), 1-19, Hermann, Paris.

[3] A. Galligo, Some algorithmic questions on ideals of differential operators, in EUROCAL '85, Vol. 2 (Linz, 1985), 413-421, Lecture Notes in Comput. Sci., 204, Springer, Berlin.

[4] M. Kashiwara, On the maximally overdetermined system of linear differential equations. I, Publ. Res. Inst. Math. Sci. 10 (1974/75), 563-579.

[5] M. Kashiwara and T. Kawai, On holonomic systems of microdifferential equations. III. Systems with regular singularities, Publ. Res. Inst. Math. Sci. 17 (1981), no. 3, 813-979. 
[6] H. Komatsu, Projective and injective limits of weakly compact sequences of locally convex spaces, J. Math. Soc. Japan 19 (1967), 366-383.

[7] _ Boundary values for solutions of elliptic equations, in Proc. Internat. Conf. on Functional Analysis and Related Topics (Tokyo, 1969), 107-121, Univ. of Tokyo Press, Tokyo.

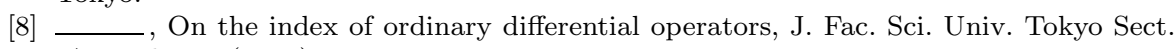
IA Math. 18 (1971), 379-398.

[9] H. Komatsu and T. Kawai, Boundary values of hyperfunction solutions of linear partial differential equations, Publ. Res. Inst. Math. Sci. 7 (1971/72), 95-104.

[10] B. Malgrange, Sur les points singuliers des équations différentielles, Enseignement Math. (2) 20 (1974), 147-176.

[11] Y. Nakamura and S. Tajima, Residue calculus with differential operator, Kyushu J. Math. 54 (2000), no. 1, 127-138.

[12] J.-P. Ramis, Dévissage Gevrey, in Journées Singulières de Dijon (Univ. Dijon, Dijon, 1978), 4, 173-204, Astérisque, 59-60, Soc. Math. France, Paris.

[13] J. T. Stafford, Module structure of Weyl algebras, J. London Math. Soc. (2) 18 (1978), no. 3, 429-442.

[14] S. Tajima, On solvability conditions for nonhomogeneous ordinary differential equations I (in Japanese). RIMS Kôkyûroku, Kyoto 1168 (2000), 66-79.

[15] _ On solvability conditions of inhomogeneous ordinary differential equations II (in Japanese), RIMS Kôkyûroku, Kyoto 1295 (2002), 9-16.

[16] Inhomogeneous ordinary differential equations and local cohomologies and residues, in Finite or infinite dimensional complex analysis and applications, 361-370, Kluwer Acad. Publ., Dordrecht.

[17] N. Takayama, kan/sm1: a computer algebra system for algebraic analysis, (1991-), http://www.math.sci.kobe-u.ac.jp/KAN/, http://www.openxm.org/ 